\title{
Paloma (RX J0524+42): the missing link in magnetic CV evolution?^
}

 \\ 1 Astrophysikalisches Institut Potsdam, An der Sternwarte 16, 14482 Potsdam, Germany \\ e-mail: rschwarz@aip.de \\ 2 Caltech Optical Observatories, Mail Code 105-24, California Institute of Technology, 1200 East California Boulevard, Pasadena, \\ CA 91125, USA \\ 3 Max-Planck Institut für extraterrestrische Physik, Postfach 1312, 85741 Garching, Germany \\ 4 Department of Physics, UC Davis, One Shields Avenue, Davis, California 95616, USA \\ 5 Observatoire Astronomique, UA 1280 CNRS, 11 rue de l'Université, 67000 Strasbourg, France
}

Received 20 April 2007 / Accepted 14 June 2007

\begin{abstract}
Decent optical photometry of the canditate magnetic CV Paloma has uncovered three persistent periods at 157, 146, and 136 min, which we interpret as the manifestation of the orbital motion of the system, the white dwarf's spin, and a related side-band frequency of the other two. All three periodicities are caused by a double-humped modulation of about 1 mag appearing only at certain fractions of the beat cycle, and it probably originates from one or two accretion spots. Our data is consistent with two plausible solutions, with the spin period being either 146 or $136 \mathrm{~min}$. The appearance of a corresponding spin-folded light curve suggests two different scenarios, for which either pole switching between two diametrically opposed accretion regions (for $P_{\text {spin }}=146$ min) or pole migration of one single spot (with $P_{\text {spin }}=136 \mathrm{~min}$ ) is the preferred accretion mode. Complementary ROSAT X-ray observations and low-resolution spectroscopy provide supporting evidence of the magnetic nature of the object. Depending on the choice of the spin period, the degree of asynchronism with respect to the orbital period is $7 \%$ or $14 \%$, implying a beat period of 0.7 or 1.4 days. Thus, the source populates the gap between the near-synchronous polars $(<2 \%)$ and the DQ Herculis stars with long spin periods (e.g. EX Hya, V1025 Cen, DW Cnc). With an orbital period right within the period gap, Paloma is a key object for magnetic CV evolution: it might be the first bona fide transition object between the DQ Her and AM Her system with a white dwarf currently in the process of synchronisation.
\end{abstract}

Key words. accretion, accretion disks - magnetic fields - X-rays: binaries - stars: novae, cataclysmic variables stars: individual: RX J0524+42

\section{Introduction}

Magnetic cataclysmic variables with an asynchronously rotating white dwarf fall into two distinct groups depending on the degree of difference between the spin of the white dwarf and the orbital motion.

First, there are the 26 confirmed DQ Herculis stars (or intermediate polars, IPs), which apart from three short orbital period systems (EX Hya, V1025 Cen and DW Cnc), host a fast spinning white dwarf with $P_{\text {spin }} \leq 0.1 \times P_{\text {orb }}$. Although there is the tendency to further subdivison the class into slow (like EX Hya) and very fast (like DQ Her itself) rotators, there is general agreement that most of the DQ Herculis stars (i) accrete via a disk (see V2400 Oph for the rare case of a diskless system; Hellier \& Beardmore 2002) and (ii) are in spin equilibrium (Norton et al. 2004). The majority of DQ Herculis stars have orbital periods longer than $3 \mathrm{~h}$, and the higher secular accretion rates expected at such long periods may represent the ultimate cause of the asynchronism. In addition, the magnetic moments of the white dwarf (although difficult to measure directly) are believed to be substantially lower than the $10^{34} \mathrm{G} \mathrm{cm}^{-3}$ that maintain the spin-orbital locking in the synchronized AM Herculis stars (or polars).

$\star$ Based in part on observations made at Observatoire de Haute Provence (CNRS), France.
The second, distinct group is formed by four nearsynchronous AM Herculis stars V1500 Cyg, BY Cam, V1432 Aql, and CD Ind, with an asynchronism - in the following defined as $\left(P_{\text {spin }}-P_{\text {orb }}\right) / P_{\text {orb }}-$ of only $\lesssim 2 \%$, they are thought to be normal AM Hers systems currently desynchronised by a recent nova explosion, as indeed observed for V1500 Cyg. Contrary to the DQ Herculis stars, these systems are believed to have accretion streams or curtains rather than disks (Schwarz et al. 2005) and are currently in the process of resynchronisation. Potentially, the near-synchronous polars are key objects to identify possible modes of asynchronous accretion (pole switching, pole migration) and measure the synchronisation torques and time scales in magnetic CVs.

In this paper we introduce the as yet uncharacterised magnetic CV RX J0524+42 or Paloma, which does not fit in either of the above categories. With a white dwarf rotating $7 \%$ or $14 \%$ faster than the binary motion and a orbital period right within the period gap, it may represent a possible transition object between the DQ Her and AM Her stars, currently in the process of synchronisation.

\section{Identification}

RX J0524+42 is an RASS X-ray point source located of only $15^{\prime}$ east of the supernova remnant VRO 42.05 .01 (Pineault et al. 1987 ) at the position $\alpha_{2000}=05^{\mathrm{h}} 24^{\mathrm{m}} 30^{\mathrm{s}} .8$ and $\delta_{2000}=42^{\circ} 44^{\prime} 53^{\prime \prime}$ 


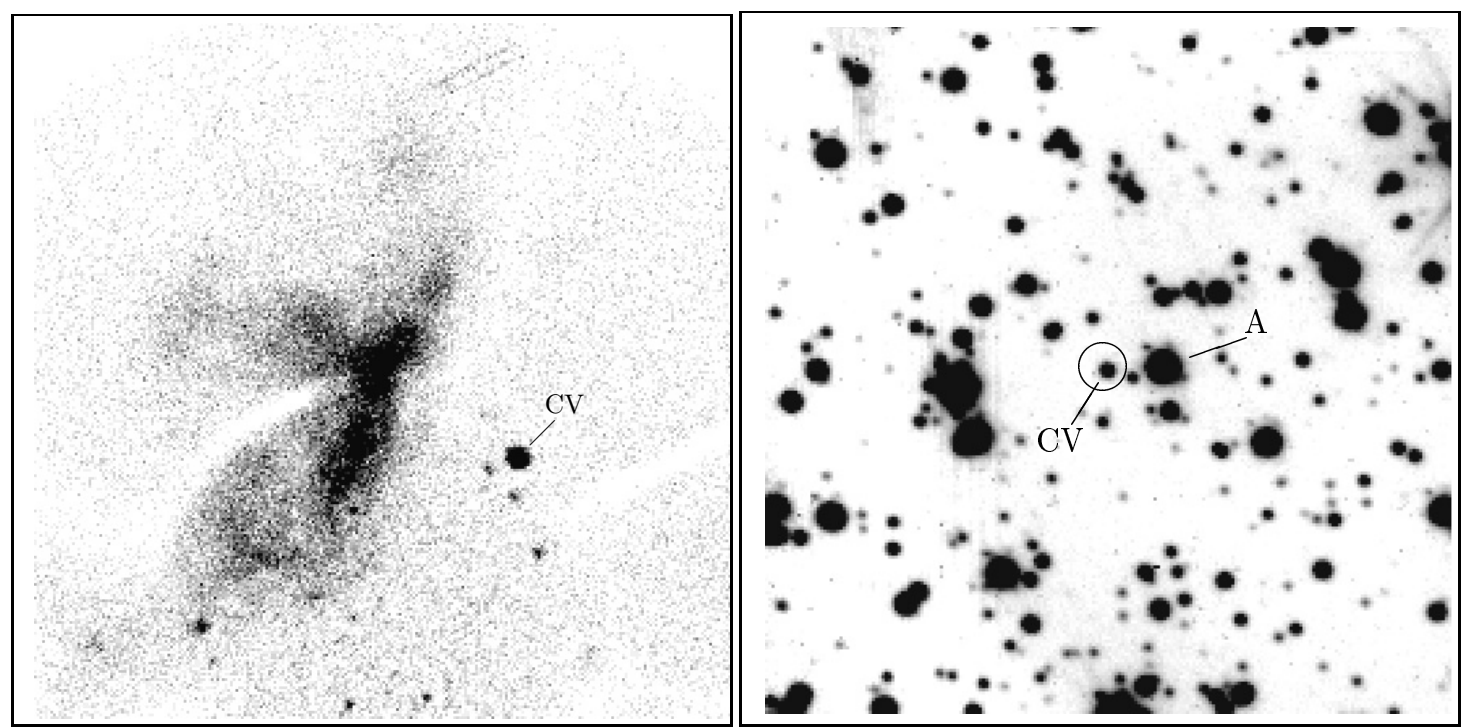

Fig. 1. Left: ROSAT X-ray image of RX J0524+42 including the SNR that inspired its name, Paloma. The field of view is about $60^{\prime} \times 60^{\prime}$ and the $\mathrm{CV}$ is the bright point source $\sim 15^{\prime}$ west of the SNR. Right: $V$-band CCD image of Paloma obtained with the AIP $70 \mathrm{~cm}$ telescope. North is on top and east to the left. The size of the field is approximately $5^{\prime} \times 5^{\prime}$. The circle refers to the $2 \sigma$ X-ray positional error. The position of the optical counterpart is $\alpha_{2000}=05^{\mathrm{h}} 24^{\mathrm{m}} 30^{\varsigma} .4$ and $\delta_{2000}=42^{\circ} 44^{\prime} 51^{\prime \prime}$.

determined in a later HRI pointing. First speculations that this source might be a kicked-out pulsar associated with the SNR were rejected after spectroscopic identification of the bright $\left(V \sim 17^{\mathrm{m}}\right)$ optical counterpart with a cataclysmic variable. Preliminary lists of ROSAT-discovered CVs tagged this source as "CV in Paloma" or short "Paloma" given the near resemblance of the SNR to a bird, a convention we will follow in this paper.

A series of follow-up spectra, taken on November 2, 1995 with the ISIS instrument at the William Herschel Telescope on La Palma, showed abundant emission lines of neutral hydrogen and helium, as well as higher excitation lines of the Bowen blend and He II $\lambda 4686$ (Fig. 2). The last two indicate a strong photonising X-ray source and are therefore tracers of magnetically channeled accretion. The integrated flux of the He II line is about one third of the $\mathrm{H} \beta$ line, which is significantly than most AM Herculis stars or the prototypical near-synchronous polar BY Cam, where it is of equal strength. During the $3 \mathrm{~h}$ of observation, the source flux smoothly varied by $1 \mathrm{mag}$, similar to what was later seen in the photometry. The blue continuum did not show obvious imprints of either the secondary star or the harmonics of a cyclotron spectrum. The absence of TiO features limits the possible contribution of the secondary in the $R$-band to $20 \%$ or $R>18$. 8 . The likely spectral type of the secondary in the period range of Paloma is M4.5 with an absolute magnitude $M_{\mathrm{R}}=11$. 89 (Kirkpatrick \& McCarthy 1994), which implies an upper limit for the distance of $240 \mathrm{pc}$.

\section{Optical photometry}

Initial CCD photometry of Paloma was obtained on January 27 and 29,1992 with the $1.2 \mathrm{~m}$ telescope equipped with the standard CCD camera of the Observatoire de Haute-Provence. Between 1995 and 2001 Paloma was observed for a total of 26 nights with the $70 \mathrm{~cm}$ telescope of the Astrophysical Institute Potsdam at Babelsberg using a cryogenic cooled $1 \times$ $1 \mathrm{k}$ TEK-CCD. The wide spacing between the individual nights caused strong alias signals in the periodograms. This was partly overcome with a dense monitoring of Paloma during another

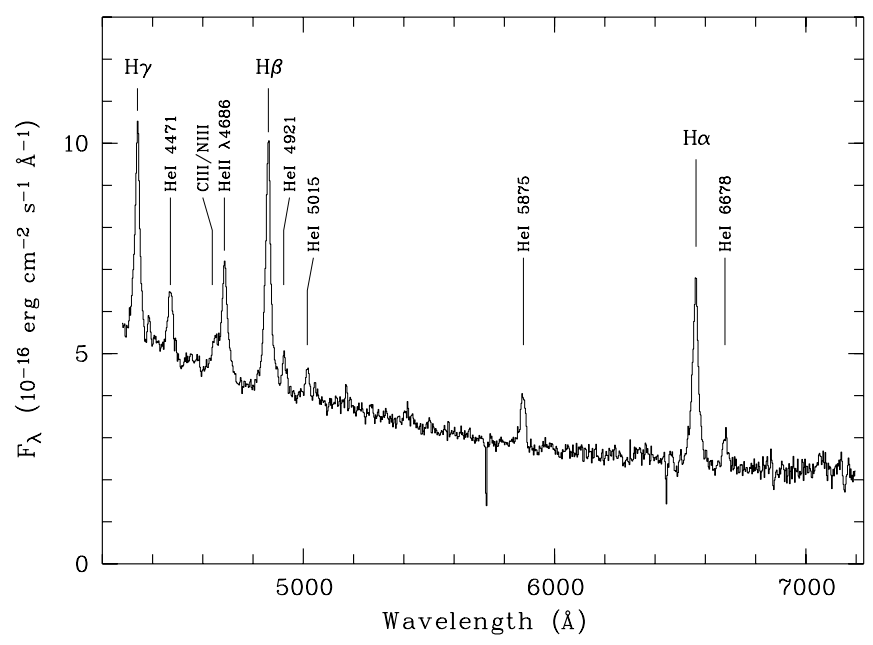

Fig. 2. Average low-resolution spectrum of Paloma derived from a series of 32 exposures taken on November 2, 1995. The flux scale has been calibrated to match the brightness level of the photometric observation on November 24, 1995. What appears as sharp absortions features are detector artefacts.

20 nights in January 2002, with both the AIP $70 \mathrm{~cm}$ reflector and the $1.23 \mathrm{~m}$ telescope at Calar Alto. Processing of the $\sim 10000$ images was done with an automated reduction pipeline based on the PSF-fitting program DoPHOT. Some of the observations were affected by clouds and fog, but first-order extinction correction was achieved by computing differential magnitudes with respect to star A (Fig. 1). On two nights, standard stars were observed from which we derived a calibrated magnitude of star A of $V=13$. 4 . The few data sets taken in the $R$ and $I$-band or without a filter were calibrated using the same zero point, therefore ignoring possible colour terms. The long-term light curve plotted in Fig. 3 shows the source at a constant mean orbital brightness level of 17 m. 5 from 1998 to early 2002 .

Initial observations suggested repetitive structures in the light curves if folded over the period of $\sim 140$ min tentatively 
Table 1. Log of photometric observations of Paloma.

\begin{tabular}{|c|c|c|c|c|c|c|c|c|c|}
\hline Date & $\begin{array}{l}T_{\text {dur }} \\
\text { (h) }\end{array}$ & Filter & $\begin{array}{r}T_{\text {int }} \\
(\mathrm{sec})\end{array}$ & $N_{\text {obs }}$ & Date & $\begin{array}{l}T_{\text {dur }} \\
\text { (h) }\end{array}$ & Filter & $\begin{array}{r}T_{\text {int }} \\
(\mathrm{sec})\end{array}$ & $N_{\text {obs }}$ \\
\hline 92 Jan. 27 & 2.7 & $V$ & 300 & 25 & 02 Jan. 2 & 12.36 & $V$ & 120 & 276 \\
\hline 92 Jan. 29 & 6.94 & $V$ & 300 & 61 & 02 Jan. 3 & 6.08 & $V$ & 120 & 165 \\
\hline 95 Nov. 24 & 4.46 & $V$ & 180 & 79 & 02 Jan. 4 & 10.30 & $V$ & 120 & 250 \\
\hline 96 Feb. 7 & 4.35 & V & 120 & 67 & 02 Jan. 5 & 3.85 & V & 120 & 97 \\
\hline 96 Feb. 8 & 3.36 & $V$ & 180 & 49 & 02 Jan. 7 & 5.29 & $V$ & 120 & 141 \\
\hline 96 Feb. 9 & 2.36 & $V$ & 180 & 44 & 02 Jan. 9 & 7.88 & $V$ & 60 & 390 \\
\hline 98 Feb. 9 & 6.65 & $W L$ & 120 & 124 & 02 Jan. 10 & 7.88 & V & 60 & 376 \\
\hline 98 Mar. 23 & 3.42 & $W L$ & 60 & 252 & 02 Jan. 11 & 7.97 & $V$ & 60 & 393 \\
\hline 98 Mar. 24 & 4.65 & $W L$ & 60 & 185 & 02 Jan. 12 & 6.98 & V & 60 & 268 \\
\hline 98 Mar. 29 & 2.89 & $V$ & 60 & 55 & 02 Jan. 14 & 12.79 & $V$ & 120 & 321 \\
\hline 98 Mar. 29 & 2.89 & $I$ & 60 & 76 & 02 Jan. 15 & 4.62 & $V$ & 120 & 174 \\
\hline 99 Nov. 3 & 4.89 & $V$ & 180 & 141 & 02 Jan. 16 & 6.91 & $V$ & 60 & 343 \\
\hline 99 Nov. 15 & 6.25 & $V$ & 120 & 172 & 02 Jan. 17 & 5.31 & $V$ & 60 & 215 \\
\hline 00 Jan. 19 & 6.21 & $V$ & 180 & 121 & 02 Jan. 18 & 4.36 & $V$ & 60 & 197 \\
\hline 00 Feb. 11 & 5.00 & $V$ & 120 & 142 & 02 Jan. 19 & 7.70 & $V$ & 60 & 386 \\
\hline 00 Feb. 21 & 2.15 & V & 120 & 59 & 02 Jan. 20 & 6.81 & $V$ & 10 & 1021 \\
\hline 00 Feb. 25 & 3.4 & V & 60 & 176 & 02 Jan. 21 & 3.21 & V & 20 & 360 \\
\hline 00 Apr. 03 & 3.95 & V & 120 & 111 & 02 Jan. 22 & 4.74 & V & 20 & 194 \\
\hline 00 Apr. 05 & 0.97 & $V$ & 120 & 22 & 02 Jan. 24 & 8.17 & $V$ & 60 & 253 \\
\hline 00 Apr. 07 & 1.92 & $V$ & 120 & 55 & 02 Jan. 25 & 6.47 & $V$ & 20 & 330 \\
\hline 00 Oct. 20 & 6.29 & $V$ & 120 & 128 & 02 Feb. 2 & 7.19 & $V$ & 60 & 381 \\
\hline 00 Nov. 16 & 3.07 & $V$ & 120 & 81 & 02 Mar. 2 & 2.26 & $V$ & 60 & 123 \\
\hline 00 Dec. 5 & 2.27 & $V$ & 120 & 64 & 02 Mar. 18 & 1.92 & $V$ & 120 & 54 \\
\hline 00 Dec. 6 & 9.01 & $W L$ & 120 & 205 & 02 Apr. 2 & 1.99 & V & 120 & 54 \\
\hline 01 Jan. 2 & 5.67 & $V$ & 120 & 161 & 02 Apr. 3 & 4.23 & $V$ & 120 & 122 \\
\hline 01 Jan. 17 & 4.54 & V & 120 & 125 & 02 Apr. 4 & 3.82 & $V$ & 60 & 105 \\
\hline 01 Apr. 2 & 3.52 & $V$ & 120 & 73 & 02 Apr. 5 & 3.67 & $V$ & 120 & 101 \\
\hline 01 Apr. 9 & 1.74 & $V$ & 120 & 47 & 02 Apr. 8 & 3.88 & $R$ & 120 & 109 \\
\hline 01 Nov. 14 & 3.32 & $V$ & 120 & 92 & & & & & \\
\hline
\end{tabular}

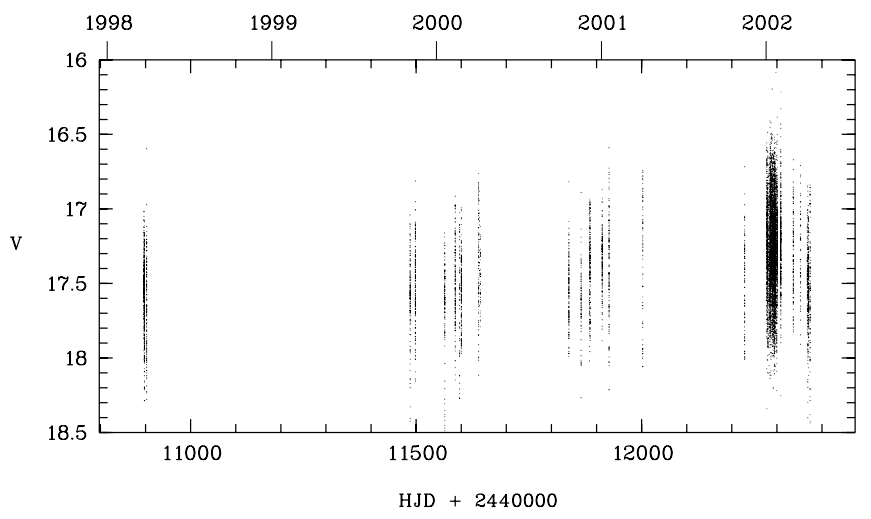

Fig. 3. Long-term light curve of Paloma.

found from these first data sets, but continuous monitoring revealed drastic nightly changes, in which the light curves (Fig. 4) appeared to show either a single asymmetric hump (Jan. 11, 2002), double humps (Nov. 15, 1999; Jan. 19, 2002) or no obvious periodic variation at all (Feb. 2, 2002).

On some occasions these slow variations were interrupted by sharp, eclipse-like features. These dips had variable width and depth and reappeared after consecutive cycles (Jan. 19, 2000; Nov. 3, 1999) or were singular events (Feb. 9, 1998). During the January 2002 campaign, the double-humped structure appeared and disappeared on a time scale of only a few days, indicating that the beat period is much shorter than in the other near-synchronous polars. Eclipse features were absent on this occasion; instead we detected single flares of 1 mag lasting a few minutes, which seemed to be fixed to certain spin phases.

\subsection{Period analysis}

In Fig. 5 we show the results of a period search using all available light curves except the scarce data before 1998. Our preferred method of period finding is the analysis-of-variance (AoV, Schwarzenberg-Czerny 1989), which is known to also have welldefined statistical properties for unevenly sampled and nonsinusoidal signals. The region of interest between 100-180 min is cluttered with a multitude of significant peaks, but one can identify three fundamental periods at $P_{1}=157, P_{2}=146$ and $P_{3}=136 \mathrm{~min}$. These are equidistant in frequency space and can be connected by a simple linear relationship $2 f_{2}=f_{1}+f_{3}$. All other remaining signals in that period range are then readily explained as half cycle/day, one cycle/day, or two cycle/day counting aliases of the fundamental periods.

Closer inspection around the main peaks (Fig. 6) reveals a fine structure on top of a broader base component. The aliases reflect the cycle-count ambiguity between the individual observing seasons, whereas the broad base component is the result of the densely spaced data set taken in January 2002. For the periods $P_{1}$ and $P_{3}$, the window pattern is dominated by a single peak, which was assigned as the true period for this paper. In the case of $P_{2}$ we adopted the value that is compatible with $P_{1}$ and $P_{3}$ by the aforementioned relationship and that coincides with the third highest peak in the periodogram. The formal accuracy of the periods, as measured from the widths (FWHM) of the peaks is $\sim 0.3 \mathrm{~s}$, does result in an uncertainty in the phase 


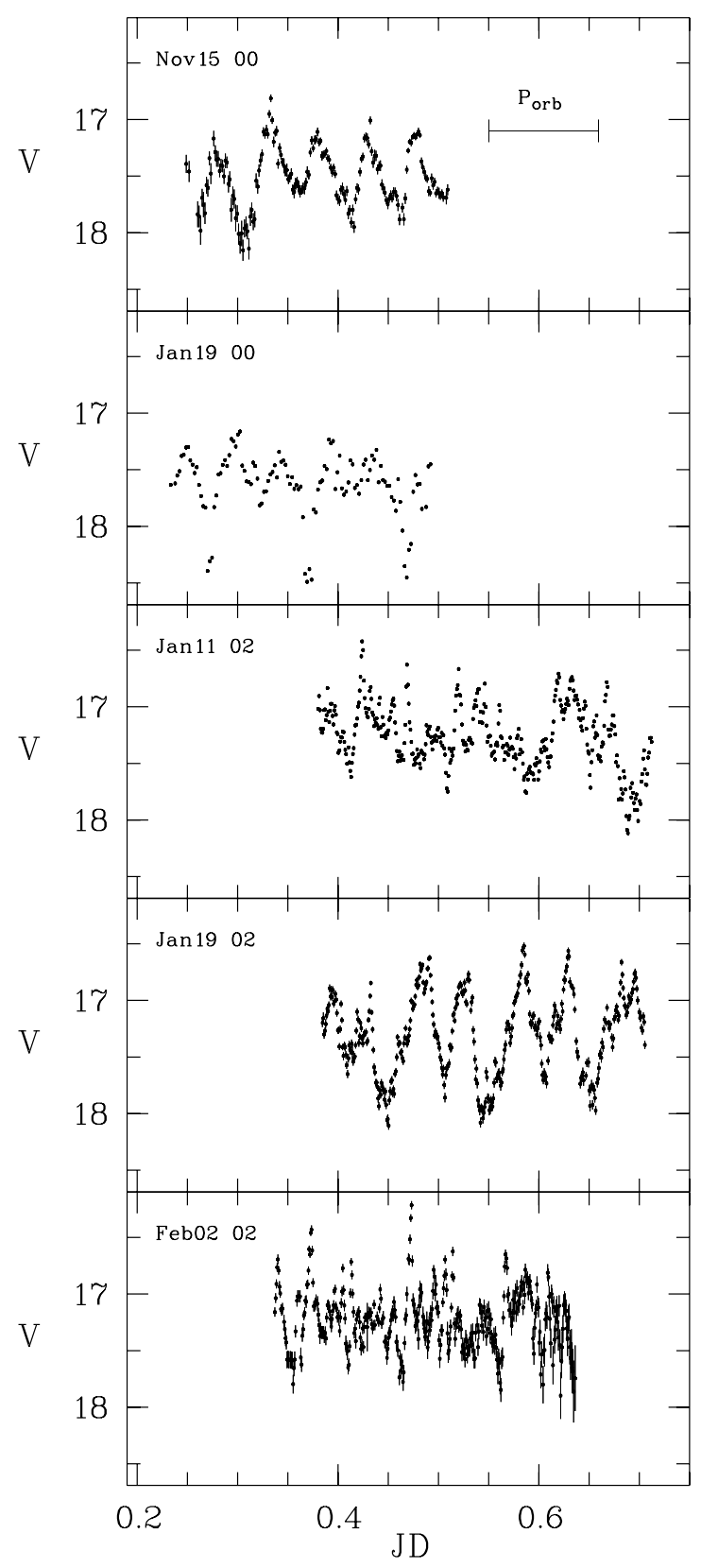

Fig. 4. Example light curves of Paloma.

folding from 1998 to 2002 of about 0.3 cycles of the orbital period. Given this, and the additional cycle count ambiguity we do not attempt to establish a long-term ephemeris. The adopted values of all prominent periodicities periods are listed in Table 2.

\subsection{Photometry around the beat cycle}

\subsubsection{Case A: $P_{\text {spin }}=146 \mathrm{~min}$}

Our photometry does not provide any a priori information on which of three main periodicities is related to the orbital motion of the system. In the following we proceed by assuming that $P_{1}$, the longest of the candidate periods at $157 \mathrm{~min}$ is indeed the orbital period $\left(P_{\text {orb }}=2 \pi / \Omega\right)$. This choice is justified by the fact that $P_{\text {orb }}>P_{\text {spin }}$ for all asynchronous magnetic CVs, except the peculiar near-synchronous system V1432 Aql
(Patterson et al. 2004), which also has the smallest difference between $P_{\text {orb }}$ and $P_{\text {spin }}$ of only $0.28 \%$.

An independent hint that the spin of the white dwarf is related to one of the shorter periods comes from the periodograms of well-sampled light curves showing the strong double-humped modulation. These data segments cover only a short fraction of the beat cycle and are much less affected by the changes of the accretion geometry, therefore better reflecting the true rotation of the accreting object. In general, these periodograms have broad peaks that are centred between 141 and 149 min with an average value of $146 \mathrm{~min}$. The close coincidence of $P_{2}$ with this value provides some evidence that it is indeed the white dwarf spin $\operatorname{period}\left(P_{\text {spin }}=2 \pi / \omega\right)$.

As a direct consequence, the signal $P_{3}$ at $136 \mathrm{~min}$ is then connected to the side-band frequency $2 \omega-\Omega$. The presence of this periodicity has been predicted for the power spectra of disk-less asynchronous magnetic CVs and can be taken as evidence of alternating accretion onto two diametrically opposed poles (Wynn $\&$ King 1992). This signal has been reported for two of the four asynchronous polars: BY Cam (Silber et al. 1997; Mason et al. 1998) and CD Ind (Ramsay et al. 1999). The detection of this signal in these systems required extensive coverage over several beat cycles, whereas smaller data sets emphasise signals near the putative spin period. Only for CD Ind is there direct evidence for the pole switching scenario by activity from different polarimetric poles occurring at particular beat phases.

Similar to Paloma, both systems show additional power at $P_{\text {spin }}$ and $P_{\text {orb }}$, the latter being a unique feature of all the diskless models of Wynn \& King (1992). This signal is the strongest of all in both CD Ind and Paloma. For the detection of $P_{2 \omega-\Omega}$ a self-eclipsing geometry with $i+\beta>90^{\circ}$ is required, a condition probably met in the case of Paloma. In the theoretical periodograms, $P_{\text {spin }}$ is absent for the case of a strict symmetry between the two accreting poles. Although, as shown later, the light curves of the two poles are indeed quite similar, the signal at $P_{\text {spin }}$ does not cancel out in the average data.

Following the above interpretation, the beat period $P_{\text {beat }}=$ $\left(P_{\text {spin }}^{-1}-P_{\text {orb }}^{-1}\right)^{-1}$ of Paloma is only 1.41 days. A signal close to that period at $2043.57 \mathrm{~min}$ is present in the AoV-periodogram (Fig. 7), but it mismatches the value actually predicted from $P_{\text {orb }}$ and $P_{\text {spin }}$ by about $4 \mathrm{~min}$. This difference is just above the error budget introduced from the two shorter periods and might therefore not be real. As an alternate and better matching interpretation, the observed value may instead represent a sub-harmonic of the spin period $\left(14 \times P_{\text {spin }}=2043.56 \mathrm{~min}\right)$ fortuitously coinciding with $P_{\text {beat }}$.

Compared to the near-synchronous polars with $P_{\text {beat }}$ in the range of 6.3 to 50 days, the much shorter beat period of Paloma provides an excellent opportunity to study photometric behaviour over the entire beat cycle. This is particularly true for the dense data set from January 2002 shown in Fig. 8, where most beat phases along a dozen consecutive beat cycles could be covered several times. The time of phase zero, as for all other cases in this paper, has been arbitrarily defined at HJD 2450897.35376 , the time of the deep optical minimum observed on March 24, 1998.

The vexing nightly behaviour is resolved into repetitive patterns, which are remarkably stable during the observations. Individual light curves taken at a certain beat phase are very similar, even though the waveform constantly changes through the beat cycle, and observations are weeks apart.

Most prominently, there is the marked rise of a doublehumped pattern with an amplitude of $\sim 1.5$ mag around two different beat phases around $\phi_{\text {beat }} \simeq 0.0$ and $\phi_{\text {beat }} \simeq 0.5$. This can 


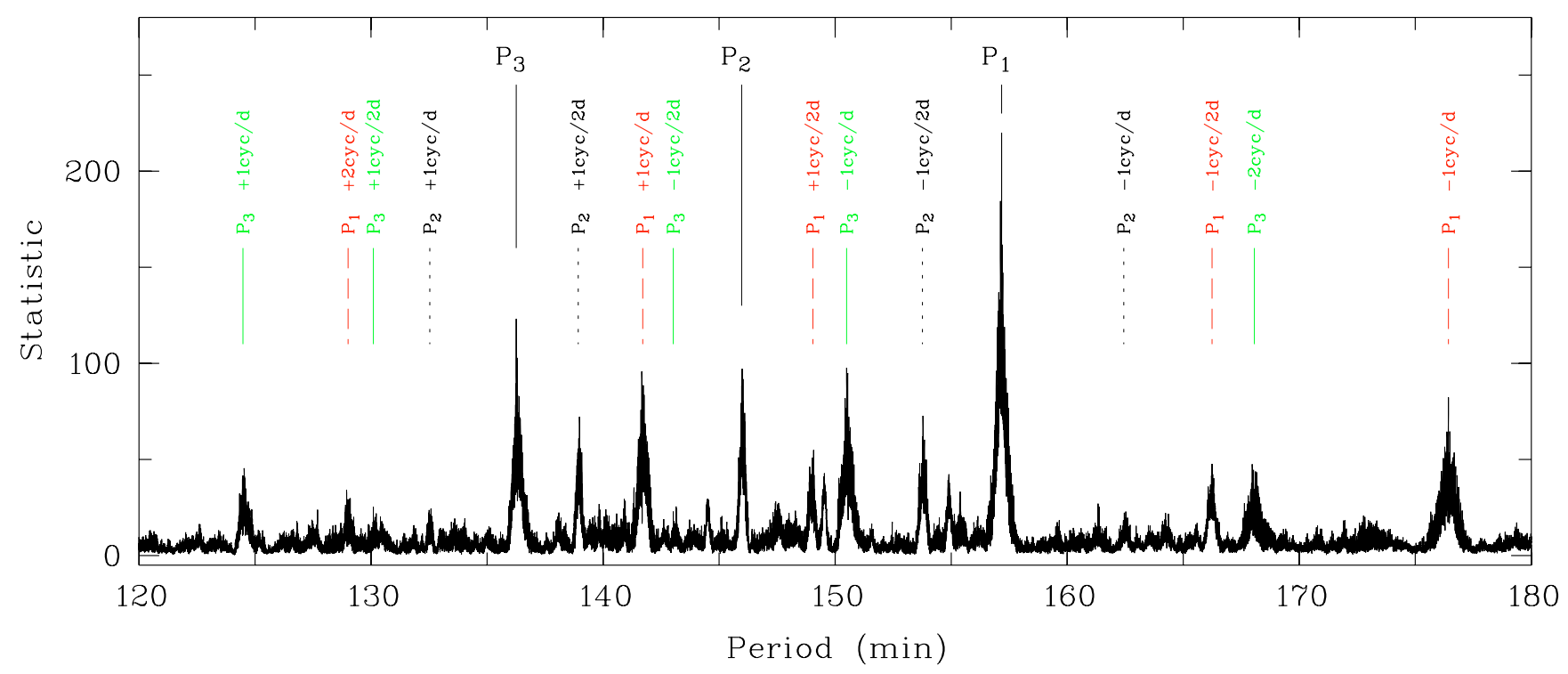

Fig. 5. AoV-periodogram of Paloma computed for photometric data obtained from 1998 to 2002 . The fundamental periods $P_{1}, P_{2}$, and $P_{3}$ and their most obvious aliases are marked. In this paper $P_{1}$ at $157 \mathrm{~min}$ is adopted as the orbital period, while the white dwarf spin can be alternatively associated with $P_{2}$ (case A) or $P_{3}$ (case B). The third periodicity is a composite of orbital and spin period and either corresponds to the $2 \omega-\Omega$ frequency (as $P_{3}=136 \mathrm{~min}$ - case A) or to the sub-harmonic of the side-band frequency $(\omega+\Omega) / 2$ (as $P_{2}=147 \mathrm{~min}-$ case B).

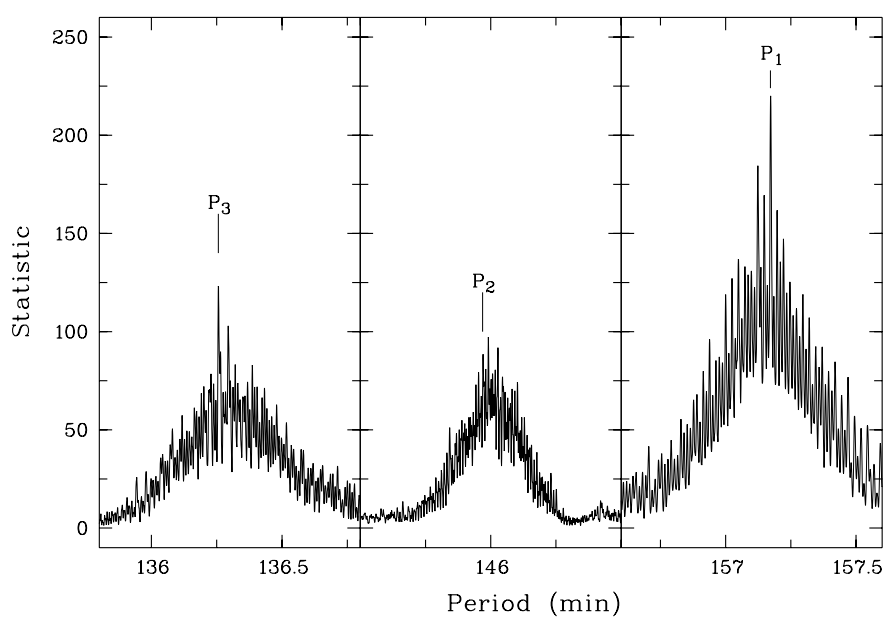

Fig. 6. Detailed view of the AoV periodogram around the three main signals.

be interpreted as alternating accretion onto two different poles. As the orientation of the field towards the infalling material constantly changes over the beat cycle, the phases of increased modulation then correspond to a situation where most of the material is predominantly transferred to one energetically preferred pole. The waveform and amplitude of the modulation itself resemble the strong cyclotron beaming observed in magnetic CVs. The interjacent intervals with much less and more random variation then likely correspond to phases where the material is transferred to both poles simultaneously, and the pole switch actually occurs.

The phases of maximum modulation are separated by $\Delta \phi_{\text {beat }} \sim 0.5$. Thus, increased funneling takes place when the magnetic axis of the white dwarf changed its orientation by $180^{\circ}$ with respect to the infalling stream, which is consistent with a dipolar field geometry.
Table 2. Significant periods detected in the combined data set of Paloma.

\begin{tabular}{|c|c|c|}
\hline Signal & Period (min) & $\begin{array}{l}\text { Identification } \\
\text { case A case B }\end{array}$ \\
\hline $\begin{array}{l}P_{1} \\
P_{2} \\
P_{3}\end{array}$ & $\begin{array}{l}157.1725 \\
145.9688 \\
136.2561 \\
78.586 \\
72.984 \\
99.681 \\
2043.576 \\
2047.752\end{array}$ & \begin{tabular}{ll}
\multicolumn{2}{c}{$P_{\text {orb }}$} \\
$P_{\text {spin }}$ & $P_{(\omega+\Omega) / 2}$ \\
$P_{2 \omega-\Omega}$ & $P_{\text {spin }}$ \\
\multicolumn{2}{c}{$P_{\text {orb }} / 2$} \\
$P_{\text {spin }} / 2$ & $P_{(\omega+\Omega)}$ \\
$P_{\omega+\Omega / 2}$ & $P_{\omega / 2+\Omega}$ \\
$P_{\text {beat }, \text { obs }}$ & $2 P_{\text {beat,obs }}$ \\
$P_{\text {beat,calc }}$ & $2 P_{\text {beat,calc }}$
\end{tabular} \\
\hline
\end{tabular}

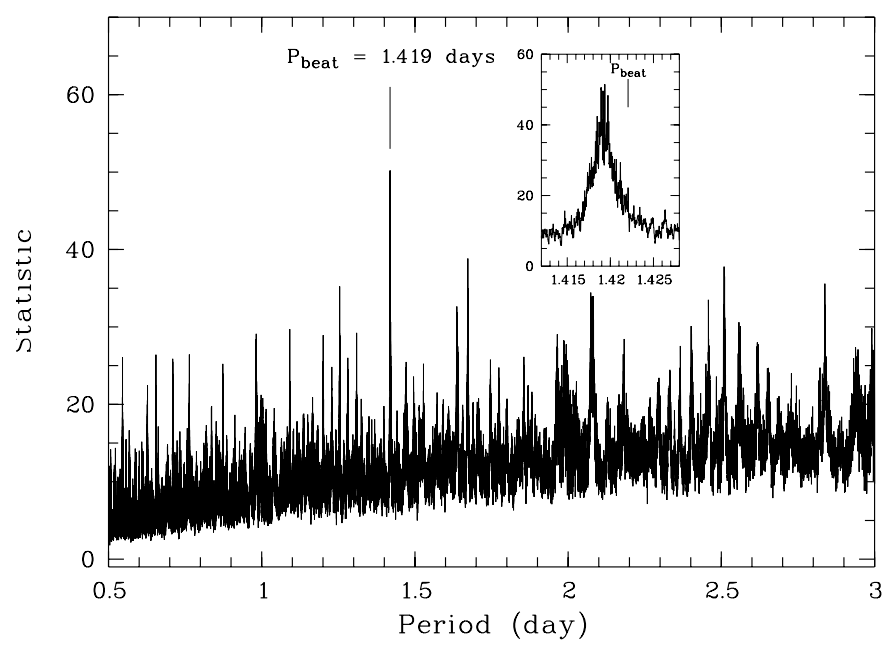

Fig. 7. AoV-periodogram of Paloma in the low frequency range showing a strong signal at 1.41915 days close to, but not exactly at the expected beat period of 1.42205 days.

A more detailed view is given by the same data set, when folded over the white dwarf's spin period and sampled into 


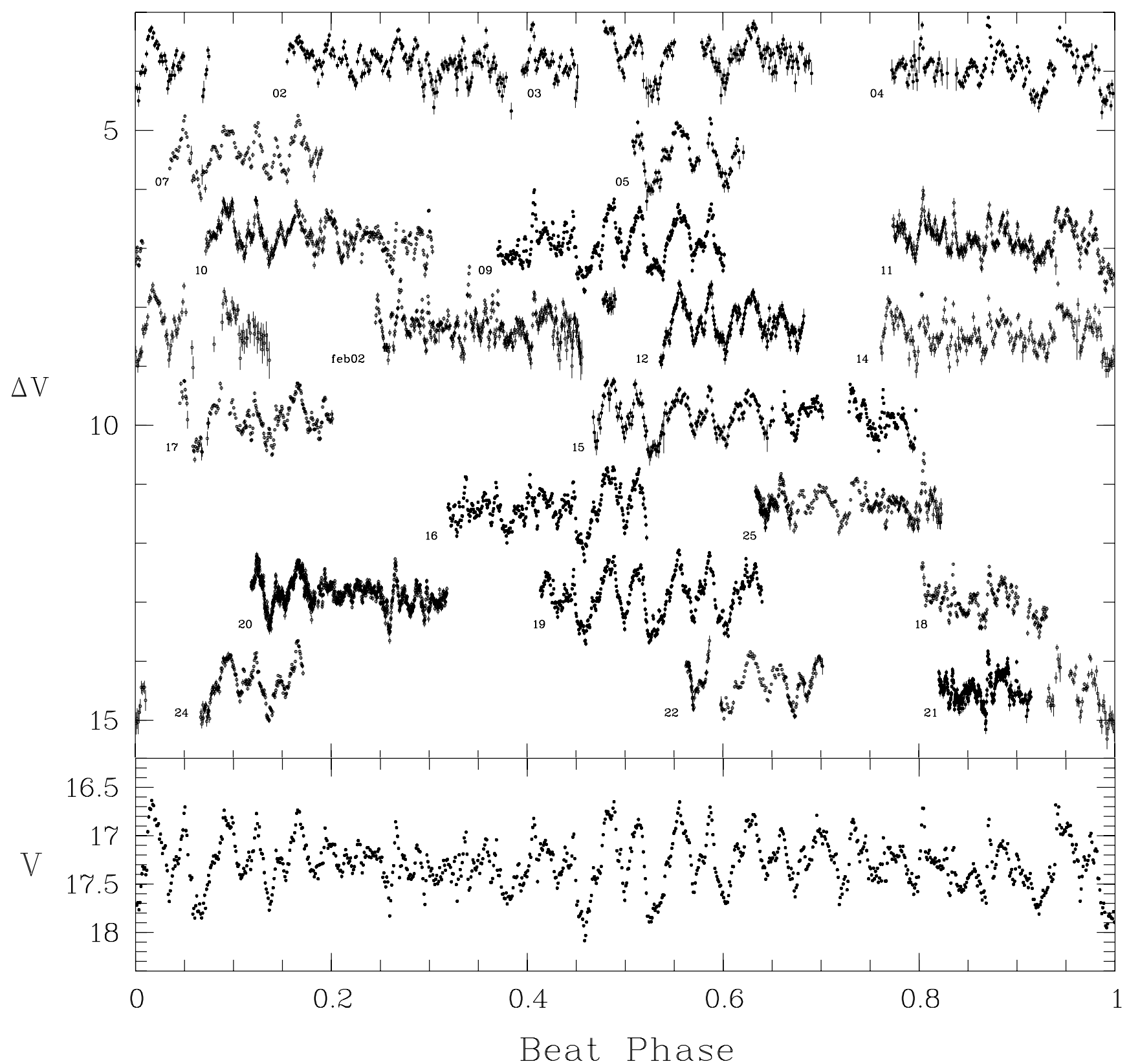

Fig. 8. Collection of all optical data taken in January 2002 folded over the beat period of 1.4 days (case A). Small numbers denote the day of month. In the lower panel the above data are phase-averaged.

individual beat phase sections (Fig. 9). The strong modulation sets in at $\phi_{\text {beat }}=0.35$ and $\phi_{\text {beat }}=0.85$, and then persists for about 3 to 4 consecutive spin cycles. It reaches maximum amplitude around $\phi_{\text {beat }}=0$ and $\phi_{\text {beat }}=0.5$. At that time the bright phases from both spots rise out of a constant minimum level suggesting a self-eclipsing accretion geometry with $\beta+i>90^{\circ}$, where $\beta$ is the colatitude of the accretion region and $i$ the inclination of the system. The duration of the bright phases of the two spots is equal to $\Delta \phi_{\text {spin }}=0.8$, indicating similar latitudes of the two emission sites. The centres of the bright phases, which measure the azimuth of the accretion regions, are at $\phi_{\text {spin }}=0$ and $\phi_{\text {spin }}=0.5$, when using the mean between start and end of the bright phase. Taking the time of the secondary minimum, which in terms of cyclotron beaming corresponds to the phase when we look most closely along the magnetic field vector, the bright phases are centred at $\phi_{\text {spin }}=0.05$ and $\phi_{\text {spin }}=0.55$. For both cases, the azimuthal offset between the two accretion spots is $180^{\circ}$, consistent with the picture that increased funnelling onto one pole occurs every half of a beat cycle.

The main difference for the beat phase intervals prior to and after the time of maximum amplitude is the duration of the bright phase, which is prolongated over the entire spin cycle. This is a hint that accretion takes place on a more extended, less focused region of the white dwarf, or that the region is shifted to lower colatitudes. The centres of the bright phase, on the other hand, seem to be fixed around $\phi_{\text {spin }}=0$ and $\phi_{\text {spin }}=0.5$. Thus, no azimuthal shift takes place with the changing beat phase.

Figure 9 also reveals interesting brightenings during the beat phase intervals that lack strong spin modulation. These features, which can be mistaken for random flaring on the larger scale of 

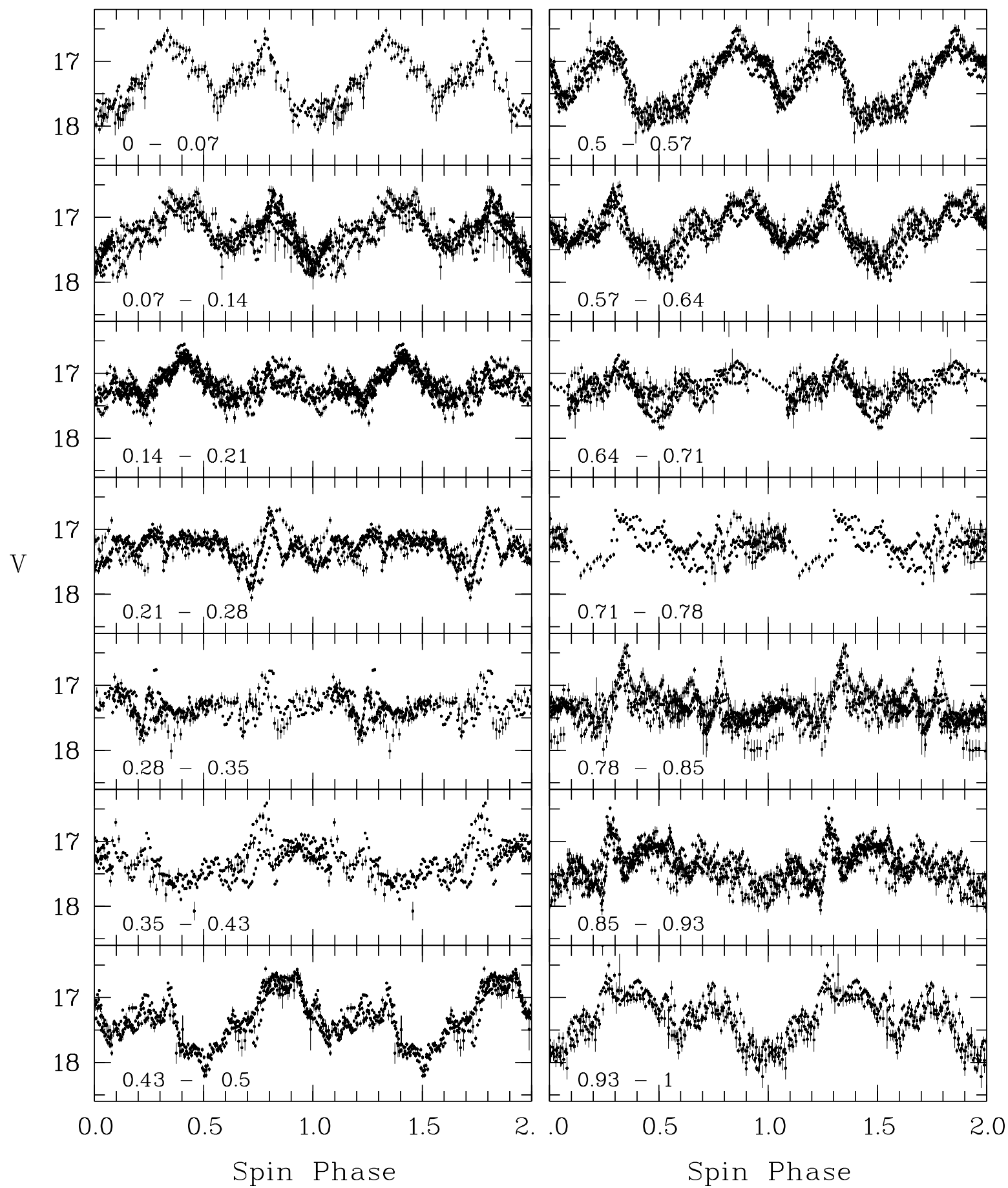

Fig. 9. Optical light curves from January 2002 as a function of spin phase separated into 14 individual sections of the 1.4 day beat period.

the beat-folded light curve (Fig. 8), are confined to certain spin phases $\left(\phi_{\text {spin }}=0.3\right.$ and $\left.\phi_{\text {spin }}=0.8\right)$. Their linear rise/decline of about $\sim 1$ mag on a time-scale as short as $5 \mathrm{~min}$ is reminiscent of non-stationary accretion of individual mass chunks, which is excluded, however, by the fact that these events reoccur at certain spin phases.

\subsubsection{Case B: $P_{\text {spin }}=136 \mathrm{~min}$}

One of the puzzling aspects of the above interpretation is the similarity of the two accretion spots. When comparing spin-folded light curves from opposite beat phases (left and right hand sides of Fig. 9) and taking the phase offset of $\Delta \phi_{\text {spin }}=0.5$ into account, the bright phases related to the two spots are indistinguishable in terms of shape, amplitude, and duration. This itself would require a highly symmetric field configuration, a distinguished viewing geometry, and nearly identical conditions in the two accretion zones.

In contrast to that, all observations of polars for which the field topology can be estimated indicate large deviations from a simple dipole. Early studies of the Zeemann lines required at least an off-centred dipole or quadrupole (Schwope et al. 1995), 
while spectropolarimetric Zeeman tomography shows evidence of higher-order multipoles (Reinsch et al. 2004). For polars where field measurements of two poles are available (Wickramasinghe \& Ferrario 2000), large differences in field strength are observed, which are difficult to reconcile with a dipole field.

Given that, it also appears plausible that the observed modulation does not come from separate spots but a single accretion region. In that case, the beat period has to be only half of what was previously assumed $\left(P_{\text {beat }}=0.7\right.$ days $)$, whereas the shortest period $P_{3}$ at $136 \mathrm{~min}$ becomes the spin period. The related period $P_{2}$ is then identified with the first sub-harmonic of the $\omega+\Omega$ side-band frequency. We note that the new beat period produces no signal in the AoV periodogram (Fig. 7). Spin and beat periods are related in such a way $\left(P_{\text {beat }} \sim 7.5 \times P_{\text {spin }}\right)$ that the strong modulation is cancelled out after one beat cycle.

In Fig. 10 we show the light curves folded over the spin period $P_{3}$ and separated into seven consecutive beat-phase intervals. The strong modulation indicating increased accretion onto one pole is observable for about half of the beat cycle between $\phi_{\text {beat }}=0.8 \ldots 1.4$. The small intrinsic scatter at a given beat interval demonstrates the close similarity of the separate spots previously assumed. As a main difference to case A, where the bright intervals were fixed to certain spin phases, we observed a systematic shift of the centre of the bright phase $\phi_{\mathrm{br}}$. We used the mean phase between two peaks of the double hump as an approximate tracer of the location of the bright phase. For the four subsequent beat-phase intervals starting at $\phi_{\text {beat }}=0.8$, we find $\phi_{\text {br }}$ at $0.53,0.61,0.71$, and 0.77 . Thus the accretion region exhibits an azimuthal shift of $80^{\circ}$ to $90^{\circ}$ over half a beat cycle. Such migration is expected if the material is threaded onto different field lines, which lead to different footpoints on the white dwarf surface. So far, such behaviour has only been detected in the asynchronous polar V1432 Aql by Geckeler \& Staubert (1997), who also provide a basic modelling of the expected phase shifts.

For a dipole configuration and a fixed threading radius, the different field lines that progressively connect to the accretion stream form an ellipse on the white dwarf. The size of the ellipse is given by the ratio of the threading and white dwarf radii, which together with the colatitude, also determines the longitudinal displacement from the magnetic axis. In Fig. 4 of Geckeler $\&$ Staubert (1997), this displacement is given as a function of the beat phase for various colatitudes and a fixed $R_{\text {thread }}=10 R_{\mathrm{wd}}$. Only for very low values $\beta<30^{\circ}$ can a large total shift as observed in Paloma be obtained. Such low colatitudes are, however, difficult to reconcile with the self-eclipsing geometry proposed for the main accretion spot. The threading radius, on the other hand, cannot be tuned to much lower values, since $10 R_{\mathrm{wd}}$ is already the radius of closest approach for a free-fall trajectory and a white dwarf of $0.6 M_{\odot}$.

The direction of the migration from earlier to later spin phases agrees with the simple picture of an oblique dipole field that changes its orientation towards the infalling gas stream. In the case of $P_{\text {spin }}<P_{\text {orb }}$, the magnetic axis passes the stagnation point in a progade direction with evolving beat phase. At earlier beat phases when accretion onto the main pole sets in, the magnetic axis lags behind the stagnation point. The footpoint of the connecting field line is therefore located at a larger azimuth $\psi^{1}$ compared to the meridian of the magnetic axis, and therefore appears at earlier spin phases. As the magnetic axis passes the

\footnotetext{
${ }^{1}$ Here we follow the standard definition for $\psi$ in polars (Cropper 1988), with $\psi$ increasing along the rotational direction in a right-handed coordinate system.
}

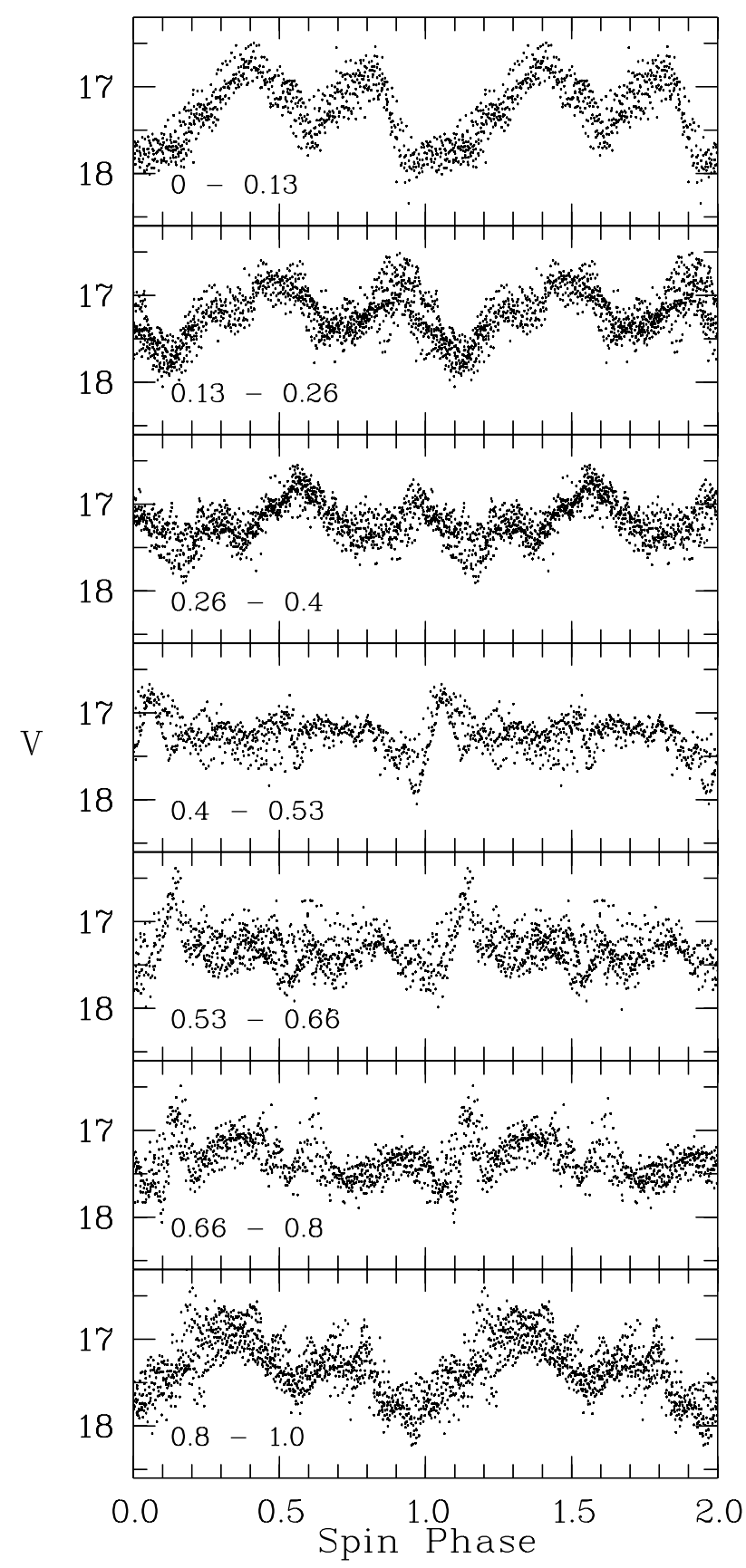

Fig. 10. Spin folded light curves from January 2002 data of Paloma now assuming $P_{3}=136 \mathrm{~min}$ as the true spin period (case B). The data are split into 7 individual sections of the beat cycle of 0.7 days.

stagnation point, field lines will lead to longitudes lagging behind the azimuth of the magnetic pole, making the bright phase then visible at later spin phases.

\section{ROSAT X-ray observations}

The ROSAT X-ray observations of Paloma comprise two PSPC pointings performed in March and September 1991, and along with another short HRI observation obtained in July 1991. Only the PSPC pointings had sufficient exposure $(17 \mathrm{ks}$ and $55 \mathrm{ks}$ ) and were considered for a spectral and temporal analysis. Extraction and binning of the X-ray photons was done with standard EXSAS routines (Zimmermann et al. 1994) including 


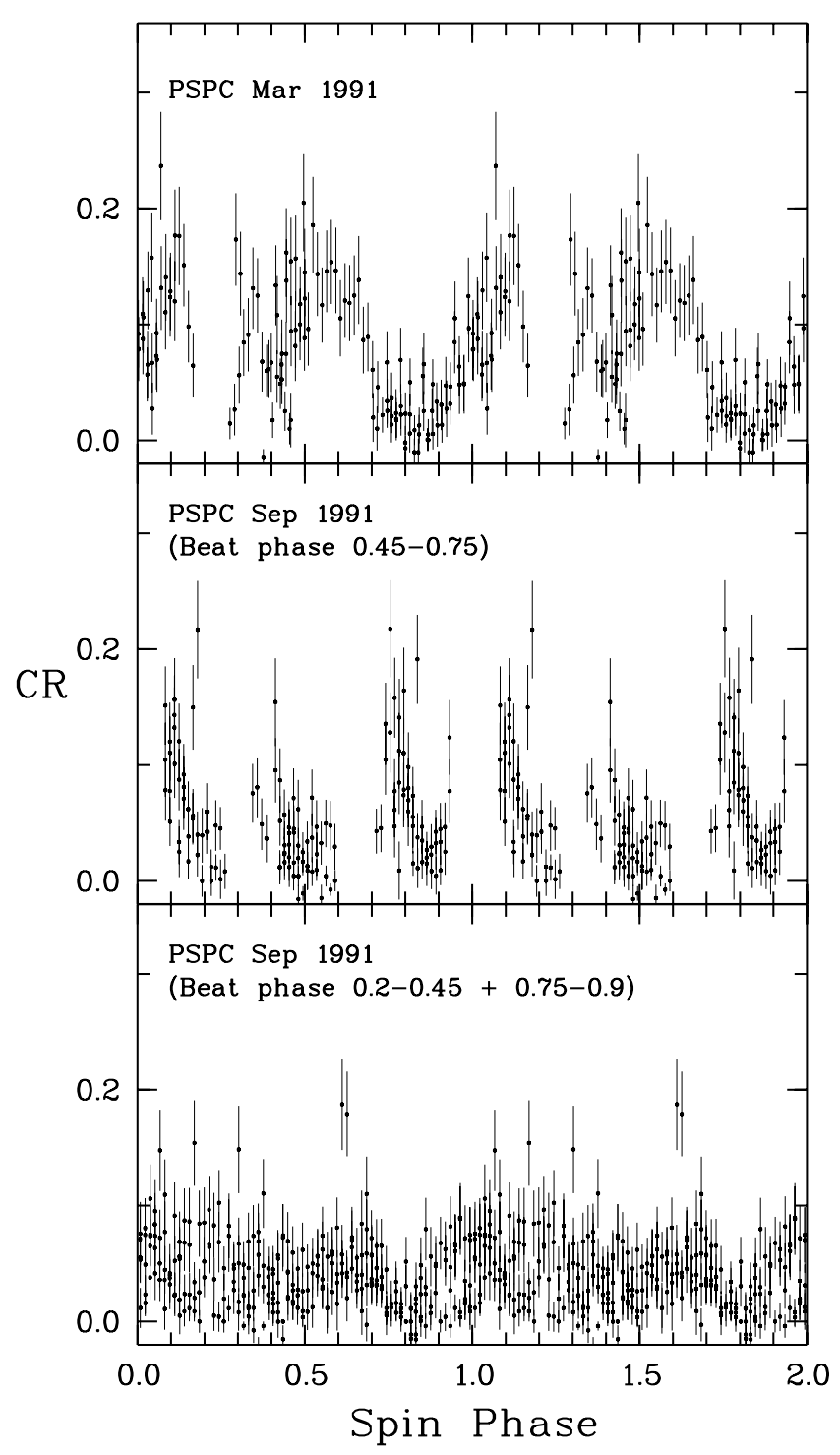

Fig. 11. X-ray light curves of Paloma from the two ROSAT PSPC pointings. The data is phased according to the spin period $P_{2}$ (case A). The longer PSPC pointing have been splitted into two sections of different beat phase intervals.

corrections for vignetting and dead time. Background-subtracted X-ray light curves were created with a binning of $120 \mathrm{~s}$. During the observations, Paloma reached peak count rates of $0.2 \mathrm{cts} / \mathrm{s}$, with mean values of $0.092 \mathrm{cts} / \mathrm{s}$ (March 91) and $0.051 \mathrm{cts} / \mathrm{s}$ (September 91). A period search of the X-ray light curves (not shown) reveals a weak, but significant, signal at the period $P_{2}$ and the orbital period, while the $P_{3}$ period was absent. The signal was stronger during the shorter pointing in March 1991, probably due to a fortuitous sampling of similar beat phases.

In Fig. 11 we show X-ray light curves folded over the assumed spin period $P_{2}$. As already suggested by the period analysis, the observation from March 1991 exhibits the largest modulation with a clear on/off behaviour reminiscent of the selfeclipsing accretion regions normally seen in polars. The duration of the bright phase is similar to what is observed in the optical. There is also evidence of dip-like sub-structures in the bright phase, indicating a complicated accretion geometry or absorption by the accretion stream.
The longer ROSAT pointing from September 1991 lasted about one day, thus spanning a wide range of the beat phase. In addition, the coverage of the individual spin cycles was limited by repeated data gaps from earth occultations of the low earth-orbit satellite. As a consequence, it is difficult to clearly identify the spin-modulated emission from the white dwarf. To avoid merging of data from widely separated beat phases, the light curve (Fig. 11) was split into two sections of the 1.4-day beat period. A shorter interval covering one quarter of the beat period was chosen such that it contains episodes of strong X-ray variability. During that part the source again reaches peak values of $0.2 \mathrm{cts} / \mathrm{s}$, but the morphology of a potential bright phase is ill-defined due to missing phase coverage. The remaining longer section shows emission at all spin phases, but at a much lower level. This and the lack of strong variability both suggest that we cover the beat intervals where the pole switching takes place.

The X-ray spectrum of Paloma in the ROSAT range is quite hard with the hardness ratios $H R 1$ of 0.56 and 0.44 measured in March and September 1991. A single-temperature thermal bremstrahlung model fixed at $k T=20 \mathrm{keV}$, plus cold absorption to the average spectra of the longer PSPC pointing, was not acceptable $\left(\chi_{v}^{2}=1.37\right)$. Inclusion of an extra blackbody component in the model significantly reduced the $\chi_{v}^{2}$ to 0.93 . The fit required a moderate amount of cold absorption of $N_{\mathrm{H}}=1 \times 10^{21} \mathrm{~cm}^{-2}$ and a best-fit blackbody temperature of $40 \mathrm{eV}$, but both parameters are not constrained very well. The unabsorbed, bolometric fluxes of the two components implied by the best-fit values are $F_{\mathrm{bb}}=8.9$ and $F_{\mathrm{br}}=5.9$ both in units of $10^{-12} \mathrm{erg} \mathrm{cm}^{-2} \mathrm{~s}^{-1}$. Thus the ratio between the emission from the hot thermal plasma and reprocessed black-body radiation is close to unity, in agreement with the standard shock model (Lamb \& Masters 1979). In that respect, the X-ray spectra of Paloma are similar to those of the other asynchronous polars (e.g. BY Cam, Ramsay et al. 1994; V1432 Aql, Staubert et al. 1994).

The lower limit to the bolometric X-ray luminosity given by the distance estimate in Sect. 2 and using a geometric factor of $2 \pi$ is $4.7 \times 10^{31} \mathrm{erg} \mathrm{s}^{-1}$. Assuming all this luminosity is due to accretion, the mass accretion rate has to be at least $1.5 \times 10^{-11} M_{\odot} \mathrm{yr}^{-1}$, a value that is in the range of normally accreting $\mathrm{CVs}$ below the period gap.

\section{Discussion}

The set of observations presented here certainly qualifies Paloma as a new disk-less, asynchronous magnetic $\mathrm{CV}$ with rather unique properties. The data are consistent with two different solutions for the spin period with $P_{\text {spin }}$ either 136 or $146 \mathrm{~min}$. The degree of asynchronism (7\% or $14 \%$ ) detected in Paloma suggests that it is a possible transition object between the AM Herculis stars in close spin-orbit locking $(<2 \%)$ and the DQ Herculis stars.

\subsection{Paloma as a probe for different accretion modes: switching vs. migration}

Interestingly, the two likely solutions for the spin period are related to two rather different accretion scenarios. Case A with $P_{\text {spin }}=146 \mathrm{~min}$ follows the "classic" interpretation for the sideband period in asynchronous polars, where it is interpreted in terms of the $2 \Omega-\omega$ frequency. In agreement with the theoretical expectations (Wynn \& King 1992), our light curves show modulation from two diametrically opposed poles, which become alternatively active. Phasing of the modulation in the spin reference frame suggests a symmetric configuration, with the 


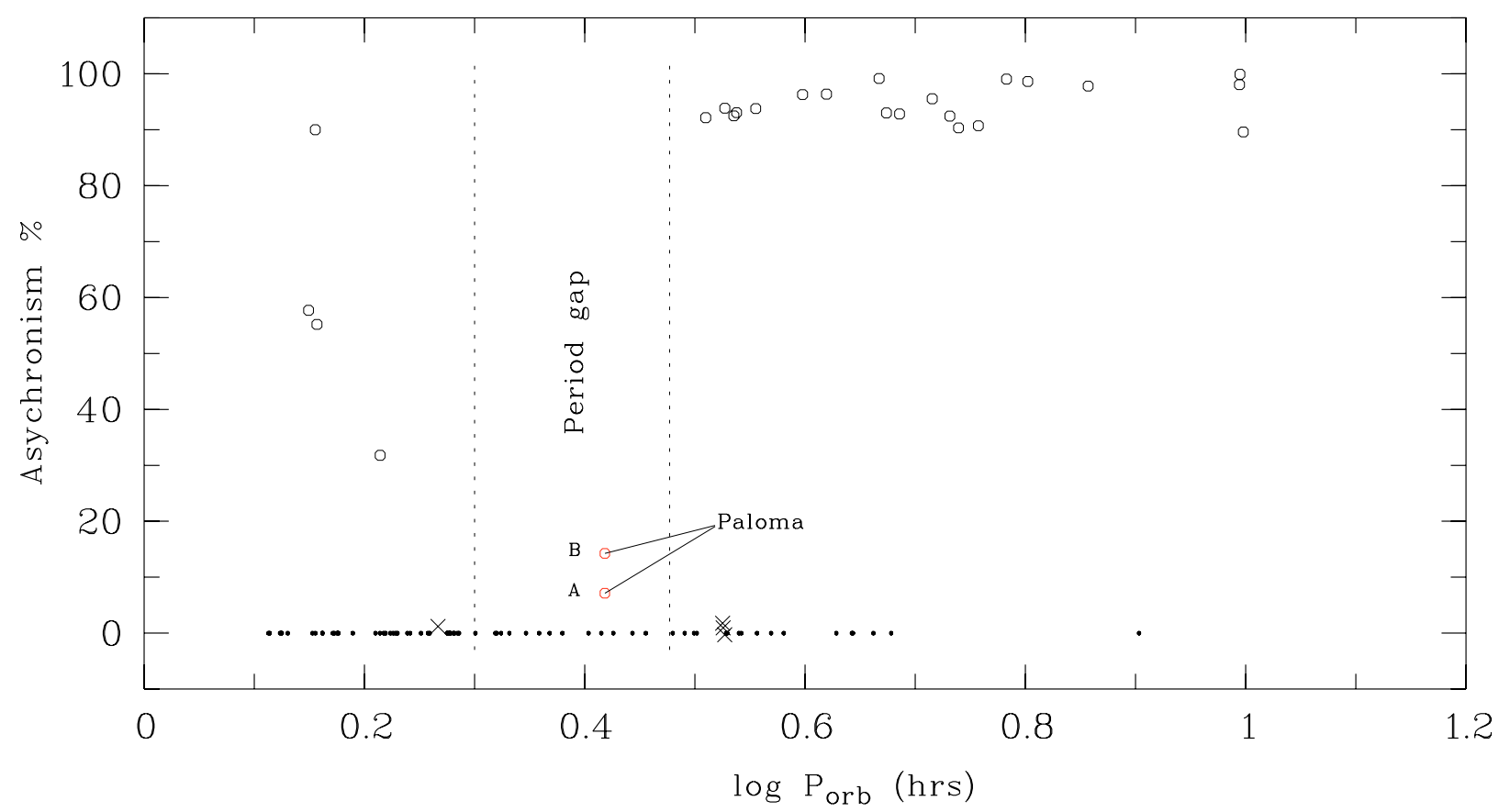

Fig. 12. The degree of asynchronism $\left(\left(P_{\text {orb }}-P_{\text {spin }}\right) / P_{\text {orb }} \times 100 \%\right)$ of magnetic CVs as a function of orbital period. The different subtypes are denoted by: DQ Herculis stars (open circles), AM Herculis stars (filled circles), and the four near-synchronous polars (crosses).

two poles separated by $180^{\circ}$. The azimuth of the poles does not change over the beat cycle. The modulation from the two spots is very similar, also requiring nearly identical latitudes and physical conditions of the accretion spots. This is also why we consider this scenario somewhat unlikely, since field configurations of accreting magnetic white dwarfs are in general much more complex.

For the alternative interpretation (case B), the shortest of the three signals previously assigned to $2 \Omega-\omega$ is the white dwarf spin period. With a beat period of now only 0.7 days, the observed modulation is related to one single accretion spot. The mass transfer rate to that pole strongly varies over the beat cycle; i.e. pole switching still takes place. However, activity from a second pole is not observable in the light curves, most likely due to disadvantageous viewing constraints. As a main difference to case $\mathrm{A}$, the main accretion region displays a large azimuthal shift of about $90^{\circ}$. The direction of the shift from earlier to later spin phases is consistent with a subsequent threading onto field lines that progradely move in the corotating binary frame. In summary, the pole migration scenario appears more plausible, although a firm decision about cases A and B can probably be obtained only by future polarimetric measurements.

The mass transfer in stream-fed, asynchronous magnetic $\mathrm{CVs}$ is far from being understood. The only object where a similar movement of the accretion region has been observed is the eclipsing system V1432 Aql. The effect there is more subtle with an amplitude of only $20^{\circ}$, but it has been independently confirmed from optical timings (Geckeler \& Staubert 1997) and X-ray eclipse measurements (Mukai et al. 2003). Contrary to Paloma, V1432 Aql shows no switching between two active regions. Most likely the situation there is complicated by a geometry with either two simultaneously accreting spots or one active pole that is always in view.

For BY Cam and CD Ind, the two systems that show similar periodicities to Paloma, one may reconsider the previous assignment of the $2 \Omega-\omega$ frequency. So far, a strong argument in favour of the pole-switching scenario in these two systems is the observation of circular polarisation of opposite sign (Piirola et al. 1994; Ramsay et al. 1999); however, the $2 \Omega-\omega$ scenario also implies peculiar accretion geometries, which might be the result of a wrong period assignment. Similar to Paloma, the light curves of BY Cam require two nearly identical poles placed at the same latitude (Mason et al. 1998), whereas in CD Ind the two poles of positive and negative polarisation appear at the same longitude (Ramsay et al. 1999). A dense polarimetric coverage over one beat cycle should clarify the ambiguous interpretations.

\subsection{Paloma in the context of $m C V$ evolution}

The evolution of magnetic CVs is, like the evolution of CVs in general, an intriguing but unresolved issue. Important information about the evolution of these systems can be drawn from the degree of synchronisation with the orbital period (Fig. 12): nearly all of the DQ Her stars ${ }^{2}$ have orbital periods above the period gap, while the majority of AM Her stars are found below the gap. This fact was interpreted in the beginning as evidence for an evolutionary link between the two classes, in which DQ Herculis stars evolve into polars objects at shorter periods (King et al. 1985). This implies that DQ Hers and AM Hers are in principle the same sort of objects, having similar magnetic field strength, and are just accreting at different mass accreting rates. Such a view was later disputed (Wickramasinghe et al. 1991) in favour of much lower field strengths for the DQ Hers, mainly based on non detecting polarised emission in most DQ Her stars. A similar conclusion is drawn from the magnetic moments theoretically derived from the conditions of spin equilibrium (Norton et al. 2004): almost all polars have magnetic moments larger

2 The sample of DQ Her stars is taken from the list of Mukai (lheawww.gsfc.nasa.gov/ mukai/iphome/iphome.html), which provides a strict, but fair, evaluation of the class membership on a semiregular basis. 
than $5 \times 10^{33} \mathrm{G} \mathrm{cm}^{3}$, whereas almost all DQ Hers lie below that value. Assigning DQ Hers and AM Hers to completely different types of objects has two uncomfortable consequences. First, there are no asynchronous high- $B$ systems at longer periods, the progenitors the AM Herculis stars, and second, we are left with no objects into which the DQ Her stars with a low magnetic field can evolve at shorter periods. The main excuse for the absence of objects in the lower right and upper left of Fig. 12 is that they are undetectable due to their peculiar emission properties.

The expanded sample of magnetic CVs found by the ROSAT mission extended the boundaries of the existing populations. There is a new class of soft X-ray DQ Hers (e.g. PQ Gem, Mason et al. 1992) that can be also polarised and does have a high enough field strength to be ancestors of the short-period AM Herculis systems. On the other hand, new short-period DQ Hers (HT Cam, Tovmassian et al. 1998; V1025 Cen, Buckley et al. 1998; and DW Cnc, Patterson et al. 2004), besides the prototypical system EX Hya $\left(P_{\text {spin }} \simeq 0.68 P_{\text {orb }}\right)$, have been discovered. All these systems show evidence of an accretion disk and are probably in spin equilibrium, thus have lower field strengths. Still missing, however, is a large quantity of systems that represent the evolutionary end points of the known DQ Her population at shorter periods.

With the discovery of Paloma, a system in the period gap, which shows a higher degree of synchronisation than any other of the three short-period DQ Hers, the old hypothesis of a close link between the two major classes of magnetic CVs is partially revived. It is one of the first good candidates for a transitional object currently in a phase of synchronisation. Such an evolutionary channel is also suggested by the recent hypothesis that the magnetic fields in IPs are buried due to the high accretion rates and resurface at shorter periods (Cumming 2002). It appears unlikely that the white dwarf of Paloma has settled into spin equilibrium. Norton et al. (2004) derived the conditions for which an asynchronous magnetic $\mathrm{CV}$ will eventually reach synchronisation. According to their estimate, all systems with $P_{\text {spin }}>0.6 P_{\text {orb }}$, irrespective of the assumed magnetic moment, will synchronise, which includes the two likely spin periods of Paloma.

The synchronisation time scales of the near synchronous polars are in the range of 100 to $1000 \mathrm{yr}$ (Campbell \& Schwope 1999). Extrapolating these values, thereby assuming similar accretion and synchronisation torques also for highly asynchronous mCVs, one finds $\tau_{\text {sync }} \sim 10^{5}-10^{6}$ yr. Comparing this synchronisation time scale with the lifetime of a CV in and above the period gap (a few $10^{8} \mathrm{yr}$ ), the probability of detecting such an object is rather low. Thus, finding one such system out of the confirmed $\sim 22$ long-period DQ Her systems seems to be inconsistent. It is suggested by King et al. (1985) that the spin evolution of highly asynchronous mCVs is determined mainly by the equilibrium of the material torques, and magnetic synchronisation becomes important only if the system is close to synchronism. The time-scale of spin evolution will be much longer in this case, coupled to the evolutionary time scale of the binary itself. In this scheme the detection of a system during synchronisation is much more plausible.

Paloma is another magnetic CV in the period gap, which accretes at a high rate, i.e. it is in an undetached state. The current sample suggests the absence of a period gap for strongly magnetic systems, although the significance of this finding is still low. A likely mechanism that explains the lack of a period gap for $\mathrm{mCVs}$ is reduced magnetic braking evoked by the interaction of the white dwarf field with the secondary's wind (Webbink \& Wickramasinghe 2002).

There is also the probability that Paloma is not crossing the gap, but has established mass transfer here for the first time. In that case, the system should be evolving from a synchronised state due to the missing accretion torque in the detached phase. This scenario requires a mass accretion rate that is much higher than normal AM Hers stars in order to break the spin-orbit locking. If not in equilibrium, the white dwarf should be currently spinning up, in contrast to an evolutionary path from the DQ Her stars.

Detection of the sign and amount of the spin-period derivative in Paloma, will provide a firm estimate of its evolutionary state and the synchronisation time scale in the more asynchronous regime.

Acknowledgements. R.S. and A.S. are supported by the Deutsches Zentrum für Luft- und Raumfahrt (DLR) GmbH under contract No. FKZ 50 OR 0404. A.S. gratefully acknowledges funding by the Deutsche Forschungsgemeinschaft under contract SCHW536/20-1.

\section{References}

Buckley, D. A. H., Cropper, M., Ramsay, G., \& Wickramasinghe, D. T. 1998, MNRAS, 299, 83

Campbell, C. G., \& Schwope, A. D. 1999, A\&A, 343, 132

Cropper, M. 1988, MNRAS, 231, 597

Cumming, A. 2002, MNRAS, 333, 589

Geckeler, R. D., \& Staubert, R. 1997, A\&A, 325, 1070

Hellier, C., \& Beardmore, A. P. 2002, MNRAS, 331, 407

King, A. R., Frank, J., \& Ritter, H. 1985, MNRAS, 213, 181

Kirkpatrick, J. D., \& McCarthy, Jr., D. W. 1994, AJ, 107, 333

Lamb, D. Q., \& Masters, A. R. 1979, ApJ, 234, L117

Mason, K. O., Watson, M. G., Ponman, T. J., et al. 1992, MNRAS, 258, 749

Mason, P. A., Ramsay, G., Andronov, I., et al. 1998, MNRAS, 295, 511

Mukai, K., Hellier, C., Madejski, G., Patterson, J., \& Skillman, D. R. 2003, ApJ, 597,479

Norton, A. J., Wynn, G. A., \& Somerscales, R. V. 2004, ApJ, 614, 349

Patterson, J., Thorstensen, J. R., Vanmunster, T., et al. 2004, PASP, 116, 516

Piirola, V., Coyne, G. V., Takalo, S. J., et al. 1994, A\&A, 283, 163

Pineault, S., Landecker, T. L., \& Routledge, D. 1987, ApJ, 315, 580

Ramsay, G., Mason, K. O., Cropper, M., Watson, M. G., \& Clayton, K. L. 1994, MNRAS, 270, 692

Ramsay, G., Buckley, D. A. H., Cropper, M., \& Harrop-Allin, M. K. 1999, MNRAS, 303, 96

Reinsch, K., Euchner, F., Beuermann, K., \& Jordan, S. 2004, in Magnetic Cataclysmic Variables, ed. S. Vrielmann, \& M. Cropper, ASP Conf. Ser., 315, IAU Colloq., 190, 71

Schwarz, R., Schwope, A. D., Staude, A., \& Remillard, R. 2005, A\&A, 444, 213 Schwarzenberg-Czerny, A. 1989, MNRAS, 241, 153

Schwope, A. D., Beuermann, K., \& Jordan, S. 1995, A\&A, 301, 447

Silber, A. D., Szkody, P., Hoard, D. W., et al. 1997, MNRAS, 290, 25

Staubert, R., Koenig, M., Friedrich, S., et al. 1994, A\&A, 288, 513

Tovmassian, G. H., Greiner, J., Kroll, P., et al. 1998, A\&A, 335, 227

Webbink, R. F., \& Wickramasinghe, D. T. 2002, MNRAS, 335, 1

Wickramasinghe, D. T., \& Ferrario, L. 2000, PASP, 112, 873

Wickramasinghe, D. T., Wu, K., \& Ferrario, L. 1991, MNRAS, 249, 460

Wynn, G. A., \& King, A. R. 1992, MNRAS, 255, 83

Zimmermann, H. U., Becker, W., Belloni, T., et al. 1994, in MPE Rep., 257 